\title{
Gravitational self-force and gauge transformations
}

\author{
Leor Barack \\ Albert-Einstein-Institut, Max-Planck-Institut für Gravitationsphysik, Am Mühlenberg 1, D-14476 Golm, Germany \\ Amos Ori \\ Department of Physics, Technion-Israel Institute of Technology, Haifa 32000, Israel
}

(Received 16 July 2001; published 31 October 2001)

\begin{abstract}
We explore how the gravitational self-force (or "radiation reaction" force), acting on a pointlike test particle in curved spacetime, is modified in a gauge transformation. We derive the general transformation law, describing the change in the self-force in terms of the infinitesimal displacement vector associated with the gauge transformation. Based on this transformation law, we extend the regularization prescription by Mino et al. and Quinn and Wald (originally formulated within the harmonic gauge) to an arbitrary gauge. Then we extend the method of mode-sum regularization (which provides a practical means for calculating the regularized self-force and was recently applied to the harmonic-gauge gravitational self-force) to an arbitrary gauge. We find that the regularization parameters involved in this method are gauge-independent. We also explore the gauge transformation of the self-force from the harmonic gauge to the Regge-Wheeler gauge and to the radiation gauge, focusing attention on the regularity of these gauge transformations. We conclude that the transformation of the self-force to the Regge-Wheeler gauge in Schwarzschild spacetime is regular for radial orbits and irregular otherwise, whereas the transformation to the radiation gauge is irregular for all orbits.
\end{abstract}

DOI: 10.1103/PhysRevD.64.124003

PACS number(s): 04.25.-g, 04.30.Db, 04.70.Bw

\section{INTRODUCTION}

Recent works by Mino, Sasaki, and Tanaka [1] and by Quinn and Wald [2] (MSTQW) established a formal framework for calculating the local gravitational self-force acting on a pointlike particle in curved spacetime. In these works, a particle of small mass $m$ was considered, whose gravitational field may be treated as a small perturbation to the (vacuum) background metric. Such a finite-mass particle does not follow a geodesic of the background geometry, as its interaction with its own gravitational field gives rise to the exertion of a "self-force." In the above works, a general formal expression was obtained for the $O(m)$ self-force correction to the geodesic equation of motion.

From the astrophysical point of view, the pointlike particle model and the self-force phenomenon may be applicable to binary systems with an extreme mass ratio. Of particular relevance are binary systems composed of a solarmass compact object orbiting a supermassive black hole (of the kind now believed to reside in the cores of many galaxies). Such systems are expected to serve as main targets for the proposed space-based gravitational wave detector LISA (the Laser Interferometer Space Antenna), specializing in the low frequency range below $1 \mathrm{~Hz}$ [3]. Knowing the local self-force would be necessary, in general, for describing the orbital evolution in such systems, and, eventually, for characterizing the consequent waveform of the gravitational radiation emitted.

When considering a model of a pointlike particle, one unavoidably encounters divergent quantities: the perturbed metric diverges at the location of the particle, and the "bare" self-force associated with the metric perturbation turns out indefinite. One then has to deal with the fundamental issue of regularization; namely, extracting the correct, physical selfforce from the (indefinite) expression for the bare self-force.
The combined works by MSTQW present three different physically motivated methods of regularization, all yielding the same formal expression for the physical self-force $F_{\text {self }}^{\alpha}$. This expression can be written in the schematic form ${ }^{1}$

$$
F_{\text {self }}^{\alpha}=F_{\text {bare }}^{\alpha}-F_{\text {inst }}^{\alpha} .
$$

Here $F_{\text {bare }}^{\alpha}$ is the "bare" force, derived by applying a certain differential operator [see Eq. (21) below] to the full metric perturbation produced by the particle, and $F_{\text {inst }}^{\alpha}$ is the singular piece to be removed. According to MSTQW analyses, this singular piece is to be constructed from the local, "instantaneous" part of the metric perturbation in the harmonic gauge, i.e., the part directly propagated along the light cone. The finite difference $F_{\text {bare }}^{\alpha}-F_{\text {inst }}^{\alpha}$ represents the effect of the "tail" part of the particle's gravitational perturbation-the part scattered off spacetime curvature before interacting back with the particle. [The result by MSTQW is formulated in terms of the retarded Green's function. The bare force is then expressed as an integral (of a certain combination of Green's function derivatives) along the entire worldline of the particle, while the instantaneous part $F_{\text {inst }}^{\alpha}$ arises from integration along an infinitesimal, local piece of the worldline, that contains the momentary particle's location.]

The first direct implementation of MSTQW's prescription for an actual calculation of the self-force was carried out

\footnotetext{
${ }^{1}$ Strictly speaking, both quantities on the right-hand side of Eq. (1) are indefinite as they stand. In practice, one actually defines these two quantities as vector fields in the neighborhood of the particle. Then, the self-force $F_{\text {self }}^{\alpha}$ is obtained by taking the (well defined and finite) limit of the difference $F_{\text {bare }}^{\alpha}-F_{\text {inst }}^{\alpha}$ as the particle is approached. For simplicity, we shall not use here this more strict formulation.
} 
recently by Pfenning and Poission [4], who considered the motion of a particle in a weakly curved region of spacetime (Pfenning and Poission also calculated the electromagnetic and scalar self-forces acting on a particle endowed with electric or scalar charges, respectively). To allow calculation of the gravitational self-force in strong field as well, Barack [5] recently introduced a method of multipole mode decomposition, based on the formal result by MSTQW. This method of "mode sum regularization" was previously developed [6] and tested [7] for the toy model of the scalar self-force. We comment that a different mode-sum approach to the gravitational self-force was proposed by Lousto [8].

The gravitational self-force-unlike its electromagnetic or scalar counterparts-is a gauge-dependent entity. This statement means that the value of the self-force is changed, in general, when the metric perturbation to which it corresponds is being subject to a gauge transformation (i.e., an infinitesimal coordinate transformation). If fact, the selfforce can be nullified along any segment of the worldline by a suitable choice of the gauge. Thus, any expression for the self-force would be meaningless, unless one is provided with the information about the gauge to which this force corresponds. In MSTQW's analysis, the construction of the selfforce is formulated within the harmonic gauge, and the resulting expression (1) therefore describes the harmonic gauge self-force. Likewise, all implementations of MSTQW's analysis considered so far [4,5] have been confined to the framework of the harmonic gauge, and have yielded the harmonic gauge self-force.

It is of great importance to understand the gauge dependence of the self-force and to figure out how to construct it in gauges other than the harmonic: From the theoretical point of view, characterization of the self-force's gauge dependence is essential for a better understanding of the self-force phenomenon; from the practical point of view, the harmonic gauge is not the most convenient one for actual calculations, as in this gauge, perturbation theory has not been developed so far to the extent it has in other gauges: In the Schwarzschild case, most analyses of metric perturbations have been formulated so far within the Regge-Wheeler gauge $[9,10]$ (see, however, the recent mode decomposition of Schwarzschild's metric perturbations in the harmonic gauge [5]). In the Kerr case, so far the only practical approach for calculating the (modedecomposed) metric perturbations is Chrzanowski's method [12], which is based on the radiation gauge.

The main purpose of this paper is to provide a general prescription for calculating the gravitational self-force in various gauges. To this end we shall first construct the general transformation law describing the behavior of the selfforce under a gauge transformation. Based on this transformation law, we re-express MSTQW's result (1) in an arbitrary gauge. We then re-formulate our method of mode sum regularization for a general gauge.

The transformation rule describing the gauge transformation of the self-force guarantees that the self-force will be well-defined if (i) it was regular in the original gauge, and (ii) the gauge transformation is sufficiently regular (namely, the displacement vector $\xi^{\mu}$ is sufficiently regular at the particle's location). A priori there is no guarantee that the trans- formation from the harmonic gauge to another desired gauge will satisfy this regularity criterion. One of the objectives of this paper is to explore the regularity of the self-force in two commonly used gauges: the Regge-Wheeler gauge and the radiation gauge. We find that the gauge transformations from the harmonic to these two gauges do not satisfy the required regularity criterion. As a consequence, our general transformation law does not yield a definite expression for the selfforce in these two gauges (the exception is the situation of a radial orbit in a Schwarzschild background, in which case the Regge-Wheeler self-force is well defined). We note that this irregularity of the gauge transformation has been noticed independently by Mino [11].

This paper is arranged as follows. We start in Sec. II by exploring the way the gravitational self-force transforms under a general gauge transformation. In Sec. III, which is somewhat out of the main course of our discussion, we consider the gauge transformation of linear gravitational forces in general. We find that this transformation law conforms with that of the gravitational self-force. The general selfforce transformation law is then used in Sec. IV to generalize MSTQW's expression for the regularized self-force from the harmonic gauge to an arbitrary gauge. We also re-formulate our method of mode sum regularization for a general gauge. A few examples are provided in Sec. V, where we consider the transformation of the self-force from the harmonic gauge to the Regge-Wheeler and to the radiation gauges. We find that in the Schwarzschild background the Regge-Wheeler self-force is well-defined for a radial orbit, but is ill-defined for non-radial orbits. The situation with the radiation gauge is even worse: It is ill-defined even for a static test particle in flat space, and hence presumably also in all types of orbit in Schwarzschild or Kerr spacetimes. Finally in Sec. VI we summarize our main results and conclusions. We also discuss the indefiniteness of the self-force in the Regge-Wheeler and radiation gauges, and suggest preliminary ways to overcome this difficulty.

Throughout this paper we use metric signature $(-+++)$ and geometrized units $G=c=1$.

\section{GAUGE TRANSFORMATION OF THE SELF-FORCE}

Our first goal in this section is to clarify the origin of the gauge dependence of the gravitational self-force. Once this origin is well understood, the derivation of transformation law for the self-force becomes rather straightforward.

In discussing the origin of the gauge dependence, we find it useful to take the following point of view towards the gravitational self-force kinematics: A point-like particle moves on a background metric $g_{0}$ (e.g., the Schwarzschild geometry), and we wish to describe the particle's orbit. The particle, having a mass $m$, deforms the geometry, which is now described by the new metric, $g=g_{0}+h$, where $h$ denotes the linearized metric perturbation produced by the particle. We also know that generally the particle will not follow a geodesic of $g_{0}$, due to its finite mass $m$. Since no external force is assumed to be present, one might attempt the simple point of view, according to which the particle moves on a "geodesic of the perturbed metric g." This naive formula- 
tion, however, is unsatisfactory (if not totally meaningless), because the perturbed metric $g$ is singular at the particle's location. We therefore must apply a different framework for analyzing the particle's motion: Assume that on the perturbed spacetime the particle follows a worldline $x^{\mu}(\lambda)$, where $\lambda$ is an arbitrary monotonous parameter (we do not assume that $\lambda$ is a proper time in $g$, because the latter is not defined, due to the divergence of $h$ ). We now project the worldline $x^{\mu}(\lambda)$ onto the background metric $g_{0}$ on the basis of "same coordinate values." [We presume here that a choice of a coordinate system has been made in advance in each of the two spacetimes. Furthermore, we assume that the coordinates in the two spacetimes are "the same" if the small perturbation is ignored-which is equivalent to assuming that $h$ is small, i.e., $O(m)$.] The projection defines a worldline $x^{\mu}(\lambda)$ on the background metric $g_{0}$, and we denote by $\tau$ the proper time along this worldline (with respect to the metric $\left.g_{0}\right)$. This construction now provides us with a natural definition of the self-force: It is simply given by the acceleration associated with the worldline $x^{\mu}(\tau)$ in $g_{0}$, through Newton's second law:

$$
F_{\mathrm{self}}^{\alpha} \equiv m\left(\frac{d^{2} x^{\alpha}}{d \tau^{2}}+\Gamma_{\mu \nu}^{\alpha}(x) \frac{d x^{\mu}}{d \tau} \frac{d x^{\nu}}{d \tau}\right)
$$

In this expression, the connection $\Gamma$ (just like the proper time $\tau)$ is taken with respect to the background metric $g_{0}$.

The origin of the gauge dependence of the self-force is now obvious: Since $g$ and $g_{0}$ represent different geometries, in principle there is no unique way to project a point (or a worldline) from $g$ to $g_{0}$. In the above formulation-as well as throughout this work-we adopt the rule of "same coordinate values." Suppose now that an infinitesimal gauge transformation is carried out in the perturbed geometry $g$, associated with an infinitesimal displacement vector $\xi^{\mu}$ :

$$
x^{\mu} \rightarrow x^{\prime \mu}=x^{\mu}-\xi^{\mu}
$$

[this transformation changes $h$ (and hence $g$ ), but of course the metric $g_{0}$ of the background spacetime is unaffected]. The particle's worldline in the perturbed spacetime now takes a new coordinate value, $x^{\prime \mu}(\lambda)=x^{\mu}(\lambda)-\xi^{\mu}$. Projecting now the worldline on $g_{0}$, one obtains a new orbit $x^{\prime \mu}\left(\tau^{\prime}\right)$, where $\tau^{\prime}$ is the proper time (in $g_{0}$ ) of the new orbit $x^{\prime \mu}(\lambda)$. It should be emphasized that the two projected worldlines, $x^{\mu}(\tau)$ and $x^{\prime \mu}\left(\tau^{\prime}\right)$, represent two physically distinct trajectories in $g_{0}{ }^{2}$ In particular, the self-force will now take a new value,

\footnotetext{
${ }^{2}$ Recall, however, that in the perturbed spacetime $g$ the two worldlines $x^{\mu}(\lambda)$ and $x^{\prime \mu}(\lambda)$ are physically equivalent-they represent the same physical trajectory in two different gauges. This difference in the relation between $x^{\mu}$ and $x^{\prime \mu}$ in the two spacetimes simply reflects the non-uniqueness of the projection from $g$ to $g_{0}$ (which, in our "same coordinate value" formulation, is tied to the arbitrariness in choosing the gauge for $h$ ).
}

$$
F_{\text {self }}^{\prime \alpha}=m\left(\frac{d^{2} x^{\prime \alpha}}{d \tau^{\prime 2}}+\Gamma_{\mu \nu}^{\alpha}\left(x^{\prime}\right) \frac{d x^{\prime \mu}}{d \tau^{\prime}} \frac{d x^{\prime \nu}}{d \tau^{\prime}}\right)
$$

where $\Gamma_{\mu \nu}^{\alpha}\left(x^{\prime}\right)$ denotes the value of the connection in the new particle's location $x^{\prime \alpha}$.

We wish to calculate the quantity $\delta F_{\text {self }}^{\alpha}$, which is the change in $F_{\text {self }}^{\alpha}$ induced by the gauge transformation, to order $m^{2}$ [recalling that $F_{\text {self }}^{\alpha}$ itself is of order $m^{2}$, and $\xi^{\mu}$ is $O(m)$ ]. To this end, we first transform the differentiation variable in Eq. (4) from $\tau^{\prime}$ to $\tau$ :

$$
\begin{gathered}
\left(\frac{d^{2} x^{\prime \alpha}}{d \tau^{\prime 2}}+\Gamma_{\mu \nu}^{\alpha}\left(x^{\prime}\right) \frac{d x^{\prime \mu}}{d \tau^{\prime}} \frac{d x^{\prime \nu}}{d \tau^{\prime}}\right) \\
=\left(\frac{d \tau}{d \tau^{\prime}}\right)^{2}\left[\frac{d^{2} x^{\prime \alpha}}{d \tau^{2}}+\Gamma_{\mu \nu}^{\alpha}\left(x^{\prime}\right) \frac{d x^{\prime \mu}}{d \tau} \frac{d x^{\prime \nu}}{d \tau}\right] \\
+\frac{d^{2} \tau}{d \tau^{\prime 2}} u^{\prime \alpha},
\end{gathered}
$$

where $u^{\prime \alpha} \equiv d x^{\prime \alpha} / d \tau$. Recalling that the term in squared brackets is already $O(m)$, we may omit the factor $\left(d \tau / \tau^{\prime}\right)^{2}$ $=1+O(\mathrm{~m})$, so at the required order we have

$$
F_{\text {self }}^{\prime \alpha}=m\left(\frac{d^{2} x^{\prime \alpha}}{d \tau^{2}}+\Gamma_{\mu \nu}^{\alpha}\left(x^{\prime}\right) \frac{d x^{\prime \mu}}{d \tau} \frac{d x^{\prime \nu}}{d \tau}\right)+\beta u^{\prime \alpha}
$$

where $\beta \equiv m\left(d^{2} \tau / d \tau^{\prime 2}\right)$. Now, the force $F_{\text {self }}^{\prime \alpha}$ must be normal to the worldline (i.e., $F_{\text {self }}^{\prime \alpha} u_{\alpha}^{\prime}=0$ ) by its definition in Eq. (4). We can therefore calculate it by projecting our last result on the direction normal to the worldline. Noting that the term $\beta u^{\prime \alpha}$ contributes nothing to this projection, we obtain

$$
F_{\text {self }}^{\prime \alpha}=m\left(\delta_{\lambda}^{\alpha}+u^{\prime \alpha} u_{\lambda}^{\prime}\right)\left(\frac{d^{2} x^{\prime \lambda}}{d \tau^{2}}+\Gamma_{\mu \nu}^{\lambda}\left(x^{\prime}\right) \frac{d x^{\prime \mu}}{d \tau} \frac{d x^{\prime \nu}}{d \tau}\right) .
$$

Rewriting $F_{\text {self }}^{\alpha}$ in the same form but with all primes omitted, and subtracting it from $F_{\text {self }}^{\prime \alpha}$ [evaluated at $x^{\prime}(x)$ ], we find at order $m^{2}$

$$
\delta F_{\mathrm{self}}^{\alpha}=m\left(\delta_{\lambda}^{\alpha}+u^{\alpha} u_{\lambda}\right)\left(q^{\prime \lambda}-q^{\lambda}\right)
$$

where

$$
q^{\prime \lambda} \equiv \frac{d^{2} x^{\prime \lambda}}{d \tau^{2}}+\Gamma_{\mu \nu}^{\lambda}\left(x^{\prime}\right) \frac{d x^{\prime \mu}}{d \tau} \frac{d x^{\prime \nu}}{d \tau}
$$

and $q^{\lambda}$ is the same but with all primes omitted. [The term proportional to $u^{\prime \alpha} u_{\lambda}^{\prime}-u^{\alpha} u_{\lambda}$ does not contribute at the relevant order, because it is itself proportional to $\xi^{\mu}$, and $q^{\lambda}$ and $q^{\prime \lambda}$ are both $O(m)$.] All we now need is to calculate $q^{\prime \lambda}$ $-q^{\lambda}$ to leading order in $\xi^{\mu}$ [expanding $\Gamma_{\mu \nu}^{\lambda}\left(x^{\prime}\right)$ about $x^{\mu}$ to leading order in $\left.\xi^{\mu}\right]$. This is a standard calculation (it is often done when constructing the Jacobi equation for geodesic deviation), and one finds 


$$
q^{\prime \lambda}-q^{\lambda}=-\left(\ddot{\xi}^{\lambda}+R_{\mu \alpha \nu}^{\lambda} u^{\mu} \xi^{\alpha} u^{\nu}\right)
$$

where an overdot denotes a covariant differentiation with respect to $\tau$ and $R^{\lambda}{ }_{\mu \alpha \nu}$ is the Riemann tensor associated with the background metric. ${ }^{3}$ Now, the term $u^{\alpha} u_{\lambda}$ in the above projection operator yields vanishing contribution when applied to the term including the Riemann tensor, due to the antisymmetry of the latter. Therefore, the final result is

$$
\delta F_{\text {self }}^{\alpha}=-m\left[\left(g^{\alpha \lambda}+u^{\alpha} u^{\lambda}\right) \ddot{\xi}_{\lambda}+R^{\alpha \lambda}{ }_{\mu \nu} u^{\mu} \xi^{\lambda} u^{\nu}\right] .
$$

(Since the calculation is carried out here at order $m^{2}$ only, in the last expression we may replace $g$ by $g_{0}$.)

The important message that arises from our discussion so far, is that the gravitational self-force is a gauge-dependent notion. Specifying $F_{\text {self }}^{\alpha}(\tau)$ by itself tells us almost nothing about the physical self-force. In order for the information on the self-force to have physical meaning, one must accompany it by the information on the gauge in which $F_{\text {self }}^{\alpha}$ was derived. Putting it in other words: The meaningful description of the gravitational self-force must include both $F_{\text {self }}^{\alpha}$ and the metric perturbation $h_{\alpha \beta}$. (Obviously, $h_{\alpha \beta}$ contains the full information about the gauge.) This is closely related to a more general feature of general-relativistic kinematics (in the non-perturbative framework): Specifying the coordinate value of a worldline $x^{\mu}(\tau)$ tells one almost nothing about the physical nature of this trajectory, unless one is also given the metric $g_{\alpha \beta}$ associated with the coordinates $x^{\mu}$.

A remark should be made here concerning the regularity of the gravitational self-force in various gauges. The construction by MSTQW yields a regular, well-defined, selfforce in the harmonic gauge. Therefore, in a given gauge $G$, the self-force will be well defined if and only if $\delta F_{\text {self }}^{\alpha}$ is well defined. Obviously, if the gauge transformation from the harmonic gauge to $G$ is defined through a perfectly regular vector field $\xi^{\lambda}$, the force in the $G$ gauge will be well defined. In most commonly used gauges, however, the vector field $\xi^{\lambda}$ associated with the transformation from the harmonic gauge to the $G$-gauge may inherit some of the irregularity that the harmonic gauge perturbation itself possesses at the particle's location (to an extent that may depend on the gauge $G$ and on the physical situation). In Sec. V this situation will be demonstrated for the RW gauge and for the radiation gauge.

A priori it is not completely obvious what degree of regularity must be imposed on $\xi^{\lambda}$ in order for the self force to be regarded as "regular." Equation (6) suggests a natural criterion for regularity: One should demand that $\xi^{\lambda}$ will be well defined (i.e. continuous) on the particle's worldline, and, furthermore, that along the worldline $\xi^{\lambda}$ will be a $C^{2}$ function of $\tau$. Note, however, that there is some arbitrariness in choosing the regularity criterion. For example, one might impose a stronger regularity criterion, which requires $\xi^{\lambda}$ to be a $C^{2}$ function of $x^{\mu}$ (such that the change in the connection due to the gauge transformation will be well defined);

\footnotetext{
${ }^{3}$ We use here the conventions of Ref. [13] for the Riemann tensor. Notice the different conventions used by Mino et al. in [1].
}

but we do not see much justification for such a strong demand. On the other hand, one may ease the above regularity criterion by extending the standard MSTQW regularization procedure and adding to it the element of averaging the selfforce (at a given moment) over all spatial directions. With this extended procedure of regularization, one may relax the demand for continuity of $\xi^{\lambda}$ at the worldline, replacing it by the weaker requirement that at the particle's location $\xi^{\lambda}$ will have a continuous limit along each spatial geodesic intersecting the worldline, and that this directional limit will be integrable over the solid angle. We further discuss this possibility at the end of the paper.

For concreteness, throughout the rest of this paper we shall adopt the criterion which naturally follows from Eq. (6) - namely, that $\xi^{\lambda}$ be continuous on the particle's worldline. The second half of this criterion-the smooth dependence on $\tau$-will automatically follow, provided that the background metric (and hence also the particle's geodesic) is sufficiently smooth, which we assume here. ${ }^{4}$

\section{GENERAL GRAVITATIONAL FORCES AND THEIR GAUGE TRANSFORMATION}

The above result (6) provides the full prescription for gauge-transforming the gravitational self-force. It will be instructive, however, to address this issue of gauge transformation from yet another point of view, by introducing the notion of a (linearized) gravitational force and studying how this force transforms in a general gauge transformation.

Consider again a spacetime described by a metric $g=g_{0}$ $+h$, where $g_{0}$ is a given background metric and $h$ denotes a linearized metric perturbation. We do not assume in this section that $h$ is a perturbation produced by a point particle; rather, $h$ is assumed to be a prescribed weak gravitational perturbation (it may represent, for example, an incident gravitational wave). Suppose that a test particle with a mass $m$ is moving freely in the perturbed spacetime. Obviously, this particle will move along a geodesic of $g$ (we neglect the self-force throughout this section ${ }^{5}$ ). Namely, we shall have, in a given coordinate system $x^{\alpha}$,

$$
\frac{d^{2} x^{\alpha}}{d \tau^{\prime 2}}+\Gamma_{\mu \nu}^{\prime \alpha} \frac{d x^{\mu}}{d \tau^{\prime}} \frac{d x^{\nu}}{d \tau^{\prime}}=0
$$

where $x^{\alpha}\left(\tau^{\prime}\right)$ denotes the particle's trajectory in the perturbed spacetime, $\tau^{\prime}$ is an affine parameter (with respect to $g$ ) along that trajectory, and $\Gamma_{\mu \nu}^{\prime \alpha}$ are the connection coefficients associated with the metric $g$. However, we now wish to take the point of view according to which the particle traces a trajectory on the background metric $g_{0}$. This trajec-

\footnotetext{
${ }^{4} \mathrm{We}$ exclude here the situation in which the gauge condition defining the $G$-gauge explicitly depends on $x^{\mu}$ or $\tau$, and this explicit dependence artificially introduces non-smoothness to $\xi^{\lambda}(\tau)$. In such spurious situations we must explicitly demand that $\xi^{\lambda}(\tau)$ be $C^{2}$.

${ }^{5}$ Throughout this section we carry out the calculation to first order in the prescribed metric perturbation $h$, and to leading order in $m$ [e.g., order $m^{0}$ in Eq. (7) below], so the self-force is not included.
} 
tory will deviate from a geodesic of the background metric $g_{0}$, and we shall interpret this deviation as representing an external "gravitational force" $F_{\text {grav }}^{\alpha}$, exerted on the particle by the perturbation $h_{\alpha \beta}$. This (fictitious) gravitational force is naturally defined as

$$
F_{\mathrm{grav}}^{\alpha} \equiv m \ddot{x}^{\alpha}=m\left(\frac{d^{2} x^{\alpha}}{d \tau^{2}}+\Gamma_{\mu \nu}^{\alpha} \frac{d x^{\mu}}{d \tau} \frac{d x^{\nu}}{d \tau}\right),
$$

where $\tau$ is an affine parameter in the background metric $g_{0}$, an overdot denotes covariant differentiation (in $g_{0}$ ) with respect to $\tau$, and $\Gamma_{\alpha \beta}^{\mu}$ are the connection coefficients associated with the metric $g_{0}$. We wish to calculate $F_{\text {grav }}^{\alpha}$ to the first order in $h$ (and to the leading order in $m$ ).

A remark should be made here concerning the relation between the gravitational self-force and the fictitious external gravitational force considered here. Obviously, the two notions are closely related, as both are defined through a mapping of a worldline from the physical spacetime $g$ to a background metric $g_{0}$. Both forces are proportional to $m$ and to the metric perturbation $h$ (though in the self-force case one assumes that $h$ is the metric perturbation produced by the particle itself). One may therefore be tempted to regard the self-force as a special case of the more general, linearized gravitational force defined here. This is not quite the case, however. The gravitational force considered here is, after all, a fictitious force; that is, the particle actually follows a geodesic of the true physical metric $g$. This cannot be said about the orbit of a particle moving under the influence of its own gravitational self-force: Since the self perturbation $h$ is singular at the particle's location, the statement that the particle follows a geodesic of $g=g_{0}+h$ is physically meaningless. ${ }^{6}$ For this reason, we must view the gravitational self force as a genuine, non-fictitious, force (though a delicate one, as expressed by its being gauge dependent).

Proceeding with the calculation of $F_{\text {grav }}^{\alpha}$, we first transform the differentiation variable in Eq. (7) from $\tau^{\prime}$ to $\tau$ [mathematically this operation is the same one applied in the previous section, Eq. (5), though here it has a somewhat different meaning]. We find

$$
\frac{d^{2} x^{\alpha}}{d \tau^{2}}+\Gamma_{\mu \nu}^{\prime \alpha} \frac{d x^{\mu}}{d \tau} \frac{d x^{\nu}}{d \tau}+\left(\frac{d \tau^{\prime}}{d \tau}\right)^{2} \frac{d^{2} \tau}{d \tau^{\prime} 2} \frac{d x^{\alpha}}{d \tau}=0
$$

Denoting $\Delta \Gamma_{\mu \nu}^{\alpha} \equiv \Gamma_{\mu \nu}^{\prime \alpha}-\Gamma_{\mu \nu}^{\alpha}$ and $u^{\alpha} \equiv d x^{\alpha} / d \tau$, and substituting Eq. (9) in Eq. (8) (keeping only terms linear in $h$ ), we obtain

$$
F_{\text {grav }}^{\alpha}=-m \Delta \Gamma_{\mu \nu}^{\alpha} u^{\mu} u^{\nu}-\beta u^{\alpha} .
$$

\footnotetext{
${ }^{6}$ One may take the point of view that the orbit of a particle under its gravitational self-force is a geodesic in a spacetime with a metric $g_{0}+h_{\text {tail }}$, where $h_{\text {tail }}$ denotes the tail part of the metric perturbation. This is, however, a fictitious geodesic, because the actual metric is $g_{0}+h$, not $g_{0}+h_{\text {tail }}$. (Recall also that in general $h_{\text {tail }}$ fails to be a vacuum solution of the linearized Einstein equations.)
}

We now get rid of the term $\beta u^{\alpha}$ by projecting $F_{\text {grav }}^{\alpha}$ on the subspace normal to $u^{\alpha}$, in the same way we treated $\delta F_{\text {self }}^{\alpha}$ above (recalling, again, that by definition $F_{\text {grav }}^{\alpha}$ is normal to $\left.u^{\alpha}\right)$. This yields

$$
F_{\text {grav }}^{\alpha}=-m\left(\delta_{\lambda}^{\alpha}+u^{\alpha} u_{\lambda}\right) \Delta \Gamma_{\mu \nu}^{\lambda} u^{\mu} u^{\nu} .
$$

Expressing $\Delta \Gamma$ in terms of $h$, we finally find

$$
F_{\text {grav }}^{\alpha}=-\frac{1}{2} m\left(g^{\alpha \lambda}+u^{\alpha} u^{\lambda}\right)\left(h_{\lambda \mu ; \nu}+h_{\lambda \nu ; \mu}-h_{\mu \nu ; \lambda}\right) u^{\mu} u^{\nu} .
$$

This expression (like the similar expressions below) is valid to linear order in the perturbation $h$, and on its right-hand side we may replace $g^{\alpha \lambda}$ by $g_{0}^{\alpha \lambda}$. It may also be useful to express Eq. (12) in terms of the trace-reversed metric perturbation $\bar{h}_{\alpha \beta} \equiv h_{\alpha \beta}-\frac{1}{2} g_{\alpha \beta} h$ (where $h \equiv g^{\alpha \beta} h_{\alpha \beta}$ ). One easily obtains

$$
F_{\text {grav }}^{\alpha}=m k^{\alpha \beta \gamma \delta} \bar{h}_{\beta \gamma ; \delta},
$$

where $k^{\alpha \beta \gamma \delta}$ is a tensor given by

$$
\begin{aligned}
k^{\alpha \beta \gamma \delta}= & \frac{1}{2} g^{\alpha \delta} u^{\beta} u^{\gamma}-g^{\alpha \beta} u^{\gamma} u^{\delta}-\frac{1}{2} u^{\alpha} u^{\beta} u^{\gamma} u^{\delta}+\frac{1}{4} u^{\alpha} g^{\beta \gamma} u^{\delta} \\
& +\frac{1}{4} g^{\alpha \delta} g^{\beta \gamma} .
\end{aligned}
$$

Next we investigate how this gravitational force is modified by a general gauge transformation (3). The metric perturbation $h$ transforms according to

$$
h_{\alpha \beta} \rightarrow h_{\alpha \beta}^{\prime}=h_{\alpha \beta}+\delta h_{\alpha \beta},
$$

where

$$
\delta h_{\alpha \beta}=\xi_{\alpha ; \beta}+\xi_{\beta ; \alpha} .
$$

From Eq. (12), the change in $h$ will induce a corresponding change in the gravitational force $F_{\text {grav }}^{\alpha}$, given by

$$
\begin{aligned}
\delta F_{\text {grav }}^{\alpha}= & -\frac{1}{2} m\left(g^{\alpha \lambda}+u^{\alpha} u^{\lambda}\right)\left(\delta h_{\lambda \mu ; \nu}+\delta h_{\lambda \nu ; \mu}-\delta h_{\mu \nu ; \lambda}\right) \\
& \times u^{\mu} u^{\nu} .
\end{aligned}
$$

Do the self-force $F_{\text {self }}^{\alpha}$ and the linearized gravitational force $F_{\text {grav }}^{\alpha}$ transform in the same manner? Substituting Eq. (15) for $\delta h_{\alpha \beta}$ in Eq. (16) and using the anti-commutation relation $\xi_{\mu ; \lambda \nu}-\xi_{\mu ; \nu \lambda}=\xi_{\rho} R_{\mu \lambda \nu}^{\rho}$, one obtains

$$
\begin{aligned}
\delta F_{\text {grav }}^{\alpha} & =-m\left(g^{\alpha \lambda}+u^{\alpha} u^{\lambda}\right)\left(\xi_{\lambda ; \mu \nu}+\xi_{\rho} R_{\mu \lambda \nu}^{\rho}\right) u^{\mu} u^{\nu} \\
& =-m\left[\left(g^{\alpha \lambda}+u^{\alpha} u^{\lambda}\right) \ddot{\xi}_{\lambda}+R^{\alpha}{ }_{\mu \lambda \nu} u^{\mu} \xi^{\lambda} u^{\nu}\right] .
\end{aligned}
$$

Comparing this expression to Eq. (6), we find that the two forces admit the same transformation law:

$$
\delta F_{\text {grav }}^{\alpha}=\delta F_{\text {self }}^{\alpha} .
$$


This result is not surprising, because the two types of forces share a common kinematic feature: They are both constructed through a projection of a worldline from a physical metric $g$ to a background metric $g_{0}$, and therefore they transform in the same manner.

\section{REGULARIZING THE GRAVITATIONAL SELF-FORCE IN VARIOUS GAUGES}

The method developed by MSTQW for regularizing the gravitational self-force is formulated within the framework of the harmonic gauge. This means that in Eq. (1) above, the two quantities on the right-hand side, $F_{\text {bare }}^{\alpha}$ and $F_{\text {inst }}^{\alpha}$ are to be evaluated in the harmonic gauge - and the outcome is the self-force in the harmonic gauge. We therefore rewrite this equation explicitly as

$$
F_{\text {self }}^{(\mathrm{H})}=F_{\text {bare }}^{(\mathrm{H})}-F_{\text {inst }}^{(\mathrm{H})},
$$

where the parenthetical index " $\mathrm{H}$ " denotes the harmonic gauge (for brevity we omit the tensorial index $\alpha$ here and in the equations below).

Assume now that a gauge transformation is made, from the harmonic gauge to a new gauge which we denote schematically by "G." According to the discussion in Sec. II, the self-force in the new gauge will be given by

$$
F_{\text {self }}^{(\mathrm{G})}=F_{\text {self }}^{(\mathrm{H})}+\delta F_{\text {self }}^{(\mathrm{H} \rightarrow \mathrm{G})}=\left[\delta F_{\text {self }}^{(\mathrm{H} \rightarrow \mathrm{G})}+F_{\text {bare }}^{(\mathrm{H})}\right]-F_{\text {inst }}^{(\mathrm{H})},
$$

where $\delta F_{\text {self }}^{(\mathrm{H} \rightarrow \mathrm{G})}$ is the expression given in Eq. (6), with $\xi^{\lambda}$ being the displacement vector that transforms from the harmonic gauge to the new gauge G. To evaluate the term in squared brackets, we first recall that the "bare force" is related to the trace-reversed metric perturbation through

$$
F_{\text {bare }}^{\alpha}=m k^{\alpha \beta \gamma \delta} \bar{h}_{\beta \gamma ; \delta}
$$

[see the second equality in Eq. (28) of Ref. [5]], which is expressed in terms of the metric perturbation itself as

$$
F_{\text {bare }}^{\alpha}=-\frac{1}{2} m\left(g^{\alpha \lambda}+u^{\alpha} u^{\lambda}\right)\left(h_{\lambda \mu ; \nu}+h_{\lambda \nu ; \mu}-h_{\mu \nu ; \lambda}\right) u^{\mu} u^{\nu} .
$$

Noting further that Eqs. (16) and (18) imply

$$
\delta F_{\text {self }}^{\alpha}=-\frac{1}{2} m\left(g^{\alpha \lambda}+u^{\alpha} u^{\lambda}\right)\left(\delta h_{\lambda \mu ; \nu}+\delta h_{\lambda \nu ; \mu}-\delta h_{\mu \nu ; \lambda}\right) u^{\mu} u^{\nu}
$$

we then obtain (using $h^{(\mathrm{H})}+\delta h^{(\mathrm{H} \rightarrow \mathrm{G})}=h^{(\mathrm{G})}$ )

$$
F_{\text {bare }}^{(H)}+\delta F_{\text {self }}^{(\mathrm{H} \rightarrow \mathrm{G})}=F_{\text {bare }}^{(G)} .
$$

This result has a simple interpretation in terms of the notion of "gravitational force" discussed in the previous section: (i) As was established there, the self-force and the gravitational force transform exactly in the same manner, and (ii) the "bare force" is nothing but the gravitational force associated with the full metric perturbation $h$ (produced by the particle). It then follows that the self-force and the bare force transform in the same manner. ${ }^{7}$

We conclude that in an arbitrary gauge $\mathrm{G}$ the regularized gravitational self-force is simply given by

$$
F_{\text {self }}^{(\mathrm{G})}=F_{\text {bare }}^{(G)}-F_{\text {inst }}^{(H)} \text {. }
$$

Namely, in an arbitrary gauge G, the singular piece to be subtracted from the bare force is always the instantaneous piece expressed in the harmonic gauge, and not in the gauge $\mathrm{G}$, as one might naively expect.

Our last result is of special importance: The analysis by MSTQW tells us how to calculate the physical self-force associated with the metric perturbation in the harmonic gauge. In particular, it tells us how to construct the "correct" instantaneous part of the bare force in this gauge. Our above discussion implies that even when calculating the self-force in a different gauge, the "correct" instantaneous part must still be calculated in the harmonic gauge. [The explicit construction of the instantaneous part from the harmonic gauge Green's function is described in Eq. (29) of Ref. [5]]. This harmonic-gauge-related instantaneous part is the one which captures the "correct" divergent piece to be removed from the bare force in whatever gauge. Intuitively, this special significance of the harmonic gauge may be attributed to its inherently isotropic nature: The "correct" divergent piece that should be removed from the bare force must be spatially isotropic (see, e.g., the analysis by Quinn and Wald [2]), and it is the harmonic gauge which admits this isotropic structure; other gauge conditions may introduce an artificial distortion to the singular piece.

\section{Mode-sum regularization in various gauges}

In Ref. [5] we introduced a practical calculation scheme for the gravitational self-force, based on the regularization procedure by MSTQW, which employs a multipole mode decomposition. This method of "mode sum regularization" has been formulated in Ref. [5] only within the harmonic gauge. Let us now examine how the above discussion, concerning the construction of the regularized self-force in various gauges, applies in the framework of the mode-sum scheme.

Within the mode sum scheme, the harmonic-gauge regularized gravitational self-force is given by [5]

$$
F_{\mathrm{self}}^{\alpha(\mathrm{H})}=\sum_{l=0}^{\infty}\left(F_{\text {bare }}^{\alpha l(\mathrm{H})}-A^{\alpha} L-B^{\alpha}-C^{\alpha} / L\right)-D^{\alpha},
$$

where the summation is over multipole modes $l$, and $L \equiv l$ $+1 / 2$. In this expression, $F_{\text {bare }}^{\alpha l(\mathrm{H})}$ is the contribution to the

\footnotetext{
${ }^{7}$ It should be emphasized that the physical notion of "gravitational force" introduced in the previous section is not necessary for the derivation of Eq. (23). Thus, starting from Eq. (6), one can derive Eq. (22) directly as a mathematical identity [following the same mathematical steps used above for constructing Eq. (17) from Eq. (16)], without any reference to the notion of "gravitational forces."
} 
self-force from $h_{\beta \gamma}^{l(\mathrm{H})}$, the $l$-mode of the metric perturbation in the harmonic gauge. This contribution is given by

$$
F_{\text {bare }}^{\alpha l(\mathrm{H})}=m k^{\alpha \beta \gamma \delta} \bar{h}_{\beta \gamma ; \delta}^{l(\mathrm{H})},
$$

where $\bar{h}_{\beta \gamma}^{l(\mathrm{H})}$ is the trace-reversed $h_{\beta \gamma}^{l(\mathrm{H})}$ and $k^{\alpha \beta \gamma \delta}$ is the tensor given in Eq. (14). The vectorial quantities $A^{\alpha}, B^{\alpha}, C^{\alpha}$, and $D^{\alpha}$ appearing in Eq. (25) are $l$-independent. These quantities, which we call "regularization parameters," are constructed from the $l$-modes of the instantaneous part $F_{\text {inst }}^{(H)}$, in a manner described in Ref. [5].

The prescription provided by Eq. (25) yields the "harmonic gauge" self-force. It is now possible, however, to reformulate this prescription in any other gauge " $\mathrm{G}$," using

$$
F_{\text {self }}^{\alpha(\mathrm{G})}=F_{\text {self }}^{\alpha(\mathrm{H})}+\delta F_{\text {self }}^{\alpha(\mathrm{H} \rightarrow \mathrm{G})} .
$$

Rewriting Eq. (22) as $\delta F_{\text {self }}^{\alpha}=m k^{\alpha \beta \gamma \delta} \delta \bar{h}_{\beta \gamma ; \delta}$ (where $\delta \bar{h}$ denotes the trace-reversed $\delta h$ ), and decomposing $\delta \bar{h}$ into $l$-modes, we obtain

$$
\delta F_{\text {self }}^{\alpha(\mathrm{H} \rightarrow \mathrm{G})}=\sum_{l=0}^{\infty} m k^{\alpha \beta \gamma \delta} \delta \bar{h}_{\beta \gamma ; \delta}^{l(\mathrm{H} \rightarrow \mathrm{G})} .
$$

Substituting this and Eq. (25) into Eq. (27), we find

$$
\begin{aligned}
F_{\text {self }}^{\alpha(\mathrm{G})}= & \sum_{l=0}^{\infty}\left[\left(F_{\mathrm{bare}}^{\alpha l(\mathrm{H})}+m k^{\alpha \beta \gamma \delta} \delta \bar{h}_{\beta \gamma ; \delta}^{l(\mathrm{H} \rightarrow \mathrm{G})}\right)\right. \\
& \left.-A^{\alpha} L-B^{\alpha}-C^{\alpha} / L\right]-D^{\alpha} .
\end{aligned}
$$

Using now Eq. (26), we can re-express the term in parentheses as

$$
m k^{\alpha \beta \gamma \delta} \bar{h}_{\beta \gamma ; \delta}^{l(\mathrm{H})}+m k^{\alpha \beta \gamma \delta} \delta \bar{h}_{\beta \gamma ; \delta}^{l(\mathrm{H} \rightarrow \mathrm{G})}=m k^{\alpha \beta \gamma \delta} \bar{h}_{\beta \gamma ; \delta}^{l(\mathrm{G})} \equiv F_{\text {bare }}^{\alpha l(\mathrm{G})},
$$

where $F_{\text {bare }}^{\alpha l(\mathrm{G})}$ denotes $l$-mode contribution to the "G-gauge bare force," namely, the contribution to the bare force from the mode $l$ of the (bare) metric perturbation in the G-gauge, through Eq. (20). We thus obtain the simple expression for the self-force in an arbitrary gauge "G,"

$$
F_{\mathrm{self}}^{\alpha(\mathrm{G})}=\sum_{l=0}^{\infty}\left(F_{\mathrm{bare}}^{\alpha l(\mathrm{G})}-A^{\alpha} L-B^{\alpha}-C^{\alpha} / L\right)-D^{\alpha} .
$$

We conclude that the regularization parameters $A^{\alpha}, B^{\alpha}$, $C^{\alpha}$, and $D^{\alpha}$ are independent of the gauge. This result has a simple intuitive explanation: These parameters are determined by the mode decomposition of the instantaneous piece of the metric perturbation, which-based on our above discussion-is always to be expressed in the harmonic gauge, regardless of the gauge chosen for calculating the self-force. Thus, the regularization parameters $A^{\alpha}, B^{\alpha}, C^{\alpha}$, and $D^{\alpha}$ are, in effect, gauge-independent.

It should be commented that the above discussion is valid as long as $\delta F_{\text {self }}^{\alpha(\mathrm{H} \rightarrow \mathrm{G})}$ (and hence the self-force in the gauge "G") admits a well defined finite value. As we demonstrate in the next section, in certain gauges the self-force turns out to be irregular or ill-defined. In such cases, the irregularity may enter Eq. (29) through the bare modes $F_{\text {bare }}^{\alpha l(\mathrm{G})}$ and render the sum over $l$ non-convergent.

\section{EXAMPLES}

In this section we study the transformation of the selfforce from the harmonic gauge to other, commonly used gauges, in a few simple cases. In principle, this transformation is done by first solving Eq. (15) for the gauge displacement vector $\xi^{\mu}$, and then constructing the force difference $\delta F_{\text {self }}^{\alpha}$ by using Eq. (6). We shall primarily be concerned here about the regularity of the self-force in the new gauge. As discussed in Sec. II, we shall regard the G-gauge self-force as regular if the vector field $\xi^{\mu}$ is continuous at the particle's location. If it is indeed continuous, then the self-force in the new gauge is given in Eq. (6) (the demand for a $C^{2}$ dependence on $\tau$ is automatically satisfied, as discussed in Sec. II). We begin by considering the transformation to the ReggeWheeler (RW) gauge, for radial trajectories in the Schwarzschild spacetime. Then we examine the transformation to the RW gauge for a uniform circular orbit. Finally, we examine the transformation to the (outgoing) radiation gauge, in a simple flat-space example.

\section{A. Regge-Wheeler gauge: Radial trajectories}

We consider a particle of mass $m$ moving along a strictly radial free-fall orbit on the background of a Schwarzschild black hole with mass $M \gg m$. (Of course, the motion of the particle will remain radial even under the effect of self-force, by virtue of the symmetry of the problem.) In what follows we use Schwarzschild coordinates $t, r, \theta, \varphi$ and assume, without loss of generality, that the radial trajectory lies along the polar axis, i.e., at $\theta=0$.

Let $h_{\alpha \beta}^{(\mathrm{H})}$ and $h_{\alpha \beta}^{(\mathrm{RW})}$ denote the metric perturbation produced by the above particle in the harmonic and RW gauges, respectively. The displacement vector field $\xi^{\mu}$ which transforms $h_{\alpha \beta}^{(\mathrm{H})}$ to $h_{\alpha \beta}^{(\mathrm{RW})}$ satisfies the gauge transformation equation

$$
h_{\alpha \beta}^{(\mathrm{RW})}=h_{\alpha \beta}^{(\mathrm{H})}+\xi_{\alpha ; \beta}+\xi_{\beta ; \alpha} .
$$

The symmetry of the physical setup motivates one to consider only axially symmetric even-parity metric perturbation modes. Accordingly, we shall look for solutions to Eqs. (30) which are $\varphi$-independent and also have $\xi_{\varphi}=0$.

For even-parity perturbation modes, the RW gauge conditions take the simple algebraic form [9]

$$
h_{t \theta}^{(\mathrm{RW})}=h_{r \theta}^{(\mathrm{RW})}=h_{\text {ang }}^{(\mathrm{RW})}=0,
$$

where $h_{\text {ang }} \equiv\left(h_{\theta \theta}-\sin ^{-2} \theta h_{\varphi \varphi}\right) / 2$. Imposing these conditions, the gauge transformation equation (30) yields three coupled differential equations for the three components $\xi_{t}, \xi_{r}$, and $\xi_{\theta}$ :

$$
\begin{gathered}
\xi_{t, \theta}+\xi_{\theta, t}=-h_{t \theta}^{(\mathrm{H})}, \\
\xi_{r, \theta}+\xi_{\theta, r}-(2 / r) \xi_{\theta}=-h_{r \theta}^{(\mathrm{H})},
\end{gathered}
$$




$$
\sin \theta\left(\sin ^{-1} \theta \xi_{\theta}\right)_{, \theta}=-h_{\text {ang }}^{(\mathrm{H})} .
$$

Equation (32c) can be immediately integrated with respect to $\theta$ (with fixed $t, r$ ), yielding

$$
\xi_{\theta}=-\sin \theta\left[\int_{0}^{\theta} \sin ^{-1} \theta^{\prime} h_{\mathrm{ang}}^{(\mathrm{H})} d \theta^{\prime}+\psi_{1}(r, t)\right],
$$

where $\psi_{1}$ is an arbitrary function. (As we shall discuss below, $h_{\text {ang }}^{(\mathrm{H})}$ vanishes sufficiently fast as $\theta^{\prime} \rightarrow 0$, such that the integral is well-defined at the lower limit.) Then, Eqs. (32a) and (32b) are immediately solvable, yielding

$$
\begin{aligned}
& \xi_{t}=-\int_{0}^{\theta}\left(h_{t \theta}^{(\mathrm{H})}+\xi_{\theta, t}\right) d \theta^{\prime}+\psi_{2}(r, t), \\
& \xi_{r}=-\int_{0}^{\theta}\left[h_{r \theta}^{(\mathrm{H})}+\xi_{\theta, r}-(2 / r) \xi_{\theta}\right] d \theta^{\prime}+\psi_{3}(r, t),
\end{aligned}
$$

where $\psi_{2}$ and $\psi_{3}$ are two other arbitrary functions. ${ }^{8}$

Now, in order to explore the behavior of the quantity $\delta F_{\text {self }}^{\alpha}$ corresponding to the gauge transformation $\mathrm{H} \rightarrow \mathrm{RW}$, by means of Eq. (6), one has to characterize the behavior of the vector field $\xi^{\mu}$ at the location of the particle. This requires one to first explore the behavior of the various $\mathrm{H}$-gauge metric functions appearing in Eqs. (33) and (34) at the particle's location. This task is most easily accomplished by considering the Hadamard form of the metric perturbation in the neighborhood of the particle. For the trace-reversed metric perturbation in the harmonic gauge, this form was given by Mino et al. [see Eq. (2.27) of Ref. [1]; alternatively, see Eq. (45) of Ref. [2]]: ${ }^{9}$

$$
\bar{h}_{\alpha \beta}^{(\mathrm{H})}=4 m \epsilon^{-1} u_{\alpha} u_{\beta}+O\left(\epsilon^{0}\right),
$$

where $\epsilon$ is the spatial geodesic distance to the particle's worldline (i.e., the proper length of the geodesic normal to the worldline which connects the latter to the evaluation point), and the terms included in $O\left(\epsilon^{0}\right)$ are assured to be at least $C^{1}$ functions of the coordinates at $\epsilon=0$. The metric perturbation itself is then given by

$$
h_{\alpha \beta}^{(\mathrm{H})}=4 m \epsilon^{-1}\left(u_{\alpha} u_{\beta}+g_{\alpha \beta} / 2\right)+O\left(\epsilon^{0}\right) .
$$

Since the worldline is radial (namely $u_{\theta}=u_{\varphi}=0$ ), it now follows that the metric perturbation components $h_{t \theta}^{\mathrm{H}}, h_{r \theta}^{(\mathrm{H})}$, and $h_{\text {ang }}^{(\mathrm{H})}$ appearing in Eqs. (33) and (34) all have vanishing contributions from the singular $O\left(\epsilon^{-1}\right)$ term, and are therefore all regular (i.e., at least $C^{1}$ ) on the worldline. Consequently, one can easily construct solutions for $\xi^{\mu}$, which

\footnotetext{
${ }^{8}$ The arbitrary functions $\psi_{i}$ represent a true freedom in the construction of the RW-gauge metric perturbations. This may be attributed to the freedom of specifying the monopole and dipole modes of the metric perturbation-see the discussion in Ref. [9].

${ }^{9}$ To obtain Eq. (35) from Eq. (2.27) of Ref. [1], recall that at the location of the particle we have $\bar{g}^{\mu}{ }_{\alpha}=\delta_{\alpha}^{\mu}$ and $\kappa=1$ (using the notation of [1]).
}

have regular, finite values at the particle's location: Starting from Eq. (33), we first observe (e.g., by transforming to Cartesian-like coordinates at the polar axis, and demanding axial symmetry as well as $C^{1}$ asymptotic behavior at $\theta=0$ ) that $h_{\mathrm{ang}}^{(\mathrm{H})}$ falls off at $\theta \rightarrow 0$ faster than $\theta$. As a consequence, the integral in Eq. (33), too, falls off faster than $\theta$. Thus, $\xi_{\theta}$ is regular at $\theta \rightarrow 0$, and it vanishes there like $\propto \theta$. (With the choice $\psi_{1}=0, \xi_{\theta}$ would vanish even faster than $\theta^{2}$.)

Consider next the two integrals in Eq. (34). From the above discussion it immediately follows that the two derivatives $\xi_{\theta_{2},}$ and $\xi_{\theta, r}$ vanish like $\propto \theta$ (at least)—like $\xi_{\theta}$ itself. Since $h_{t \theta}^{\mathrm{H}}$ and $h_{r \theta}^{\mathrm{H}}$ are regular $\left(C^{1}\right)$ too, we find that the two integrands in Eq. (34) are bounded at $\theta=0$. (In fact, by transforming to Cartesian-like coordinates near $\theta=0$ one can easily verify that $h_{t \theta}^{\mathrm{H}}$ and $h_{r \theta}^{\mathrm{H}}$-and hence the two integrands-vanish at $\theta \rightarrow 0$.) Consequently, the two integrals vanish at $\theta \rightarrow 0$. We find that along the particle's worldline all components of $\xi^{\mu}$ are regular, and satisfy

$$
\xi_{\theta}=0, \quad \xi_{t}=\psi_{2}(r, t), \quad \xi_{r}=\psi_{3}(r, t),
$$

where $\psi_{2}(r, t)$ and $\psi_{3}(r, t)$ are freely specifiable functions. (In fact, this holds not only at the particle's worldline, but everywhere along the polar axis.) Furthermore, choosing $\psi_{2}=\psi_{3}=0$, we obtain a solution for $\xi^{\mu}$ which is not only regular but is also vanishing along the particle's worldline: $\xi^{\mu}(\tau)=0$.

Since the above-constructed vector $\xi^{\mu}$ is continuous at the particle's location, we obtain - through Eq. (6) — a regular finite value for the desired quantity $\delta F_{\text {self }}^{\alpha}$. Thus, for strictly radial trajectories in Schwarzschild spacetime, the gravitational self-force is regular in the $R W$ gauge. Moreover, this RW-gauge self-force can be made equal to the harmonicgauge self force, by exploiting the remaining freedom in the RW gauge (manifested here by the three arbitrary functions $\left.\psi_{1-3}\right)$.

\section{B. Regge-Wheeler gauge: Circular orbits}

Let us now consider a particle which (in the lack of selfforce) moves on a circular geodesic at $r=r_{0} \geqslant 6 \mathrm{M}$ around a Schwarzschild black hole. Without loss of generality, we shall assume an equatorial orbit (i.e., $\theta=\pi / 2$ and $u^{\theta}=0$ ) and will consider the self-force at a point $\mathrm{P}$ located on the particle's orbit at $t=\varphi=0$. In this physical scenario, the metric perturbation contains both even and odd parity modes. The RW gauge condition [9] then becomes a bit more complicated than the one specified in Eq. (31) for a purely even perturbation (in general, the two algebraic conditions $h_{t \theta}^{(\mathrm{RW})}$ $=h_{r \theta}^{(\mathrm{RW})}=0$ are no longer valid, and are to be replaced by conditions involving derivatives of the metric perturbation). However, the two gauge conditions involving the angular components of the metric perturbation maintain a simple algebraic form, namely

$$
h_{\theta \varphi}^{(\mathrm{RW})}=0, \quad h_{\mathrm{ang}}^{(\mathrm{RW})}=0 .
$$

For our purpose, it will be sufficient to consider only these 
two conditions. When imposed on the gauge transformation equation (30), these conditions lead to a set of two coupled equations for $\xi_{\theta}$ and $\xi_{\varphi}$ :

$$
\begin{gathered}
\sin \theta\left(\sin ^{-1} \theta \xi_{\theta}\right)_{, \theta}-\sin ^{-2} \theta \xi_{\varphi, \varphi}=-h_{\text {ang }}^{(\mathrm{H})} . \\
\xi_{\theta, \varphi}+\sin ^{2} \theta\left(\sin ^{-2} \theta \xi_{\varphi}\right)_{, \theta}=-h_{\theta \varphi}^{(\mathrm{H})},
\end{gathered}
$$

The source terms for these equations are evaluated, again, with the help of Eq. (36): We find that $h_{\theta \varphi}^{(\mathrm{H})}$ is regular at the particle's location, but the source for Eq. (38a) diverges there as $-h_{\text {ang }}^{(\mathrm{H})} \cong a r_{0}^{2} \epsilon^{-1}$, where $a=2 m r_{0}^{-2} u_{\varphi}^{2}=2 m\left(r_{0} / M-3\right)^{-1}$ $[15]$ and, as before, $\epsilon$ denotes the spatial geodesic distance to the particle's worldline. In what follows we analyze the behavior of $\xi_{\theta}$ and $\xi_{\varphi}$ at the immediate neighborhood of $\mathrm{P}$, to leading order in $\epsilon$.

We first note that no derivatives with respect to $r$ and $t$ appear in Eq. (38a) (though the source term depends on $r$ and $t$ through $\epsilon$ ). Therefore, this equation can be solved for each $r, t$ separately. For our purpose-demonstrating the discontinuity of the solution at $\mathrm{P}$-it will be sufficient to consider the solution at the two-dimensional plain $r=r_{0}, t=0$, which is simpler to analyze.

To bring Eqs. (38) to a convenient form, we introduce the local Cartesian-like coordinates $y \equiv r_{0} \sin \theta \sin \varphi, \quad z$ $\equiv r_{0} \cos \theta$ in the neighborhood of P. Note that $z=y=0$ at $\mathrm{P}$, and that (for $r=r_{0}, t=0$ ) at the leading order we have $\epsilon$ $=\left[\left(1-v^{2}\right)^{-1} y^{2}+z^{2}\right]^{1 / 2}$. Here $v$ denotes the particle's velocity in the Lorentz frame of a static local observer, $v$ $\equiv\left(-g_{\varphi \varphi} / g_{t t}\right)^{1 / 2}(d \varphi / d t)$. One can easily obtain the explicit value of $v[15]$ :

$$
v=\left(-g^{\varphi \varphi} / g^{t t}\right)^{1 / 2}\left(u_{\varphi} / u_{t}\right)=\left(r_{0} / M-2\right)^{-1 / 2}<1 .
$$

Transforming in Eqs. (38) from $(\theta, \varphi)$ to $(z, y)$ we obtain two coupled equations for $\xi_{z}$ and $\xi_{y}$, reading

$$
\begin{gathered}
\left(1-z^{2} / r_{0}^{2}\right) \xi_{z, z}-\left(1-y^{2} / r_{0}^{2}\right) \xi_{y, y}=a / \epsilon+\cdots, \\
\xi_{z, y}+\xi_{y, z}-2 y z\left(r_{0}^{2}-z^{2}\right)^{-1} \xi_{y, y}=0+\cdots,
\end{gathered}
$$

where the dots $(\cdots)$ represent corrections to the source term which are at least $C^{1}$ at $\mathrm{P}$. As we are interested only in the leading-order behavior of $\xi^{\mu}$ at $\mathrm{P}$ [where $\left(z / r_{0}\right)^{2},\left(y / r_{0}\right)^{2},\left(z y / r_{0}^{2}\right)$ all vanish], we shall proceed by restricting attention to the leading-order form of Eqs. (39):

$$
\begin{aligned}
& \xi_{z, z}-\xi_{y, y}=a / \epsilon, \\
& \xi_{z, y}+\xi_{y, z}=0 .
\end{aligned}
$$

Equation (40b) allows us to express the vector $\xi^{\mu}$ in terms of a scalar potential $\Phi$, as ${ }^{10}$

\footnotetext{
${ }^{10}$ Defining $\vec{E} \equiv\left(E_{y}, E_{z}\right) \equiv\left(-\xi_{y}, \xi_{z}\right)$, Eq. (40b) reads $\nabla \times \vec{E}=0$, which allows one to define $\vec{E}=\nabla \Phi$. (Note, however, that since there is a singularity at $y=z=0, \Phi$ need not be single-valued-see the discussion below.)
}

$$
\xi_{z}=\Phi_{, z}, \quad \xi_{y}=-\Phi_{, y} .
$$

With Eq. (40a), this potential is then found to satisfy Poisson's equation

$$
\Phi_{, z z}+\Phi_{, y y}=a / \epsilon
$$

It is convenient to introduce polar coordinates in the $z y$-plain, which we define through $z=\rho \sin \phi, \quad y=\rho \cos \phi$. Transforming in Eq. (42) from $z, y$ to $\rho, \phi$, and substituting $\epsilon=\left[\left(1-v^{2}\right)^{-1} y^{2}+z^{2}\right]^{1 / 2}$, we obtain

$$
\rho^{-1}\left(\rho \Phi_{, \rho}\right)_{, \rho}+\rho^{-2} \Phi_{, \phi \phi}=\frac{a}{\rho}\left(1-v^{2}\right)^{1 / 2}\left(1-v^{2} \sin ^{2} \phi\right)^{-1 / 2} .
$$

Next, we wish to expand $\Phi(\rho, \phi)$ into angular Fourier modes $e^{i n \phi}$. Before doing this, however, there is a subtlety that must be discussed. The displacement vector $\xi^{\mu}$ must be a single-valued (SV) function of $\phi$. This means that both $\Phi_{, \phi}$ and $\Phi_{, \rho}$ must be SV too. However, in principle the generating potential $\Phi$ need not be a SV function of $\phi$. Therefore, in the complete mode decomposition of $\Phi$ one may also include certain functions of $\phi$ which are not necessarily SV. However, since the $\phi$-derivative of each such multi-valued function must be SV, this function must be linear in $\phi$ (such that the Fourier expansion of $\Phi_{, \phi}$ will only include SV Fourier modes). Furthermore, since the $\rho$-derivative must be SV too, this "linear mode" must be independent of $\rho$. The full decomposition thus takes the form

$$
\Phi(\rho, \phi)=c \phi+\sum_{n=-\infty}^{\infty} e^{i n \phi} \Phi_{n}(\rho),
$$

where $c$ is an arbitrary constant. Substituting this form in Eq. (43) (recalling that $c \phi$ satisfies the homogeneous part of this equation), one obtains an ordinary equation for each $n$-mode, reading

$$
\rho^{-1}\left(\rho \Phi_{n, \rho}\right)_{, \rho}-n^{2} \rho^{-2} \Phi_{n}=\frac{a}{\rho} f_{n},
$$

where the coefficients $f_{n}$ are given by the (elliptic) integrals

$$
f_{n}=\frac{\sqrt{1-v^{2}}}{2 \pi} \int_{0}^{2 \pi} \frac{e^{-i n \phi}}{\sqrt{1-v^{2} \sin ^{2} \phi}} d \phi .
$$

It can be easily verified that $f_{n}$ vanishes for all odd $n$. For even $n$, however, $f_{n}$ is generally non-vanishing. In particular, for $n=0$ the integrand in Eq. (46) is bounded from below by unity, hence $f_{0}>\sqrt{1-v^{2}}>0$.

The general exact solution to Eq. (45) is easily constructed:

$$
\Phi_{n}=\left\{\begin{array}{lll}
b_{0} \rho+\alpha_{0}+\beta_{0} \ln \rho & \text { for } & n=0, \\
b_{n} \rho+\alpha_{n} \rho^{|n|}+\beta_{n} \rho^{-|n|} & \text { for } & n \neq 0,
\end{array}\right.
$$

where $\alpha_{n}$ and $\beta_{n}$ are arbitrary constants, and 


$$
b_{n}= \begin{cases}a f_{n} /\left(1-n^{2}\right) & \text { for even } n, \\ 0 & \text { for odd } n .\end{cases}
$$

We may now construct the modes of $\xi^{\mu}$ by applying Eq. (41) to each of the single modes. We then wish to figure out what is the solution with the most regular behavior at the limit $\rho$ $\rightarrow 0$, which concerns us here. Clearly, any choice of $\beta_{n} \neq 0$ will lead to a divergent $\Phi_{n}$ and hence to a divergent vector $\xi^{\mu}$. [Note that the norm of $\left(\xi_{y}, \xi_{z}\right)$ is the same as that of $\nabla \Phi$, and is hence bounded below by $\left|\Phi_{, \rho}\right|$; and the contribution to the latter from a nonvanishing $\beta_{n}$ would diverge like $\propto \rho^{-|n|-1}$.] Similarly, a nonvanishing $c$ would yield a potential $\Phi$ whose (normalized) derivative in the tangential direction, $\rho^{-1} \Phi_{, \phi}$, diverges like $c \rho^{-1} .{ }^{11}$ The most regular solution is thus one with $\beta_{n}=0$ for all $n$, as well as $c=0$. This solution takes the form

$$
\Phi(\rho, \phi)=\alpha_{0}+\rho H(\phi)+O\left(\rho^{2}\right),
$$

where

$$
H(\phi)=\left(\alpha_{1} e^{i \phi}+\alpha_{-1} e^{-i \phi}\right)+\sum_{n=-\infty}^{\infty} b_{n} e^{i n \phi} .
$$

Returning from $\Phi$ to $\xi^{\mu}$, we find e.g., for the Cartesianlike component $\xi_{y}$ (ignoring higher-order contributions in $\rho)$ :

$$
\xi_{y}=-\Phi_{, y}=-\rho_{, y} H-\rho \phi_{, y} H_{, \phi} .
$$

Substituting $\rho_{, y}=\cos \phi$ and $\phi_{, y}=-\rho^{-1} \sin \phi$, we find

$$
\xi_{y}=-H \cos \phi+H_{, \phi} \sin \phi \equiv \xi_{y}(\phi) .
$$

Clearly, in order for $\xi_{y}$ to be continuous at $\rho \rightarrow 0$ (where $\phi$ is indefinite), it must be independent of $\phi$. However, $\xi_{y, \phi}$ $=\left(H_{, \phi \phi}+H\right) \sin \phi$, and

$$
H_{, \phi \phi}+H=\sum_{n=-\infty}^{\infty}\left(1-n^{2}\right) b_{n} e^{i n \phi}=a \sum_{n=-\infty}^{\infty} f_{n} e^{i n \phi} .
$$

This function of $\phi$ does not vanish (identically) unless all coefficients $f_{n}$ vanish; however, as was shown above, $f_{0}$ $>0$. We find that $\xi_{y}(\rho \rightarrow 0)$ does depend on $\phi$ (the same can be shown for $\xi_{z}$ ). This means that the vector $\xi^{\mu}$ is discontinuous at $\mathrm{P}^{12}$

\footnotetext{
${ }^{11}$ Divergent contributions from different $n$-modes cannot cancel each other, because they have different dependence on $\phi$, as well as different rates of divergence $\left(\rho^{-|n|-1}\right)$. Also, a divergence coming from the linear mode cannot cancel a divergent $n=0$ mode, even though in both modes $|\nabla \Phi| \propto \rho^{-1}$, because the direction of $\nabla \Phi$ is tangential for the linear mode and "radial" for the $n=0$ mode.

${ }^{12}$ The indefiniteness of the RW self-force could be intuitively understood, by realizing that the RW gauge condition "distracts," to some amount, the presumed isotropic structure of the divergent local piece of the metric perturbation, by artificially signifying the $\theta$ direction. (This isotropic structure is best accounted for within the harmonic gauge.)
}

As the gauge displacement vector $\xi^{\mu}$ does not admit a definite value at the particle's location, Eq. (6) cannot be used, as it stands, for constructing the self-force in the RW gauge. Following the discussion at the end of Sec. II, we arrive at the conclusion that in the case of circular motion, the " $R W$ self-force" is ill defined (unless one further extends the regularization procedure-e.g., by introducing an average over solid angle; see the discussion in Sec. II).

We conclude this discussion with two remarks: First, though the discontinuity of $\xi^{\mu}$ was explicitly demonstrated here for circular orbits, this conclusion should also apply to generic non-radial, non-circular, orbits (for radial orbits, however, it was demonstrated above that $\xi^{\mu}$ is continuous).

Second, the above construction shows that for a suitable choice of the free parameters (namely $c=\beta_{n}=0$ ) the component $\xi_{y}$ is bounded at $\mathrm{P}$. The same holds for $\xi_{z}$. This implies that $\xi_{\theta}$ and $\xi_{\varphi}$ are bounded (though discontinuous) at the particle's location. It still remains to be checked, however, whether $\xi_{t}$ and $\xi_{r}$ are bounded or not.

\section{Radiation gauge}

Finally, we examine the transformation of the self-force to the so-called "radiation gauge." (We recall that, so far, the mode decomposition of metric perturbations in Kerr spacetime has been formulated primarily within the radiation gauge [12].) We shall consider here the simplest possible case: a static particle in flat spacetime. As we shall shortly see, even in this trivial case, the gauge transformation from the harmonic to the radiation gauge is pathological, and the metric perturbation (and hence the self-force) is ill defined.

We shall specifically consider the outgoing radiation gauge (similar results are obtained when considering the ingoing radiation gauge). We use standard flat-space spherical coordinates $t, r, \theta, \varphi$, and assume that the static particle is located off the origin of the spherical coordinates, i.e., at some $r=r_{0}>0$. Also, without loss of generality, we locate the particle at the polar axis, $\theta=0$. The outgoing null vector field takes the form $l^{\alpha}=[1,1,0,0]$. The metric perturbation in the radiation gauge, $h_{\alpha \beta}^{(\mathrm{R})}$, is defined by the requirement ${ }^{13}$

$$
h_{\alpha \beta}^{(\mathrm{R})} l^{\beta}=0 .
$$

Consider now the $t$-component of Eq. (48), which reads

$$
h_{t t}^{(\mathrm{R})}+h_{t r}^{(\mathrm{R})}=0 .
$$

With the gauge transformation equation $h_{\alpha \beta}^{(\mathrm{R})}=h_{\alpha \beta}^{(\mathrm{H})}+\xi_{\alpha ; \beta}$ $+\xi_{\beta ; \alpha}$, this becomes

$$
\xi_{t, r}+\xi_{r, t}+2 \xi_{t, t}=-h_{t t}^{(\mathrm{H})}-h_{t r}^{(\mathrm{H})} .
$$

\footnotetext{
${ }^{13}$ In the case of a pure vacuum perturbation over a Kerr background, the additional condition $h^{(\mathrm{R})} \equiv g^{\alpha \beta} h_{\alpha \beta}^{(\mathrm{R})}=0$ can be imposed in a consistent manner, as done by Chrzanowski in [12]. Here we consider the perturbation in a region surrounding a point source, and it is unclear to us whether the additional condition $h^{(\mathrm{R})}=0$ will be consistent with the gauge condition (48). We shall therefore not make any use of this extra condition here.
} 
Motivated by the staticity of the problem, we shall only consider $t$-independent solutions, so $\xi_{r, t}$ and $\xi_{t, t}$ may be dropped. Also, in the harmonic gauge we have

$$
h_{t t}^{(\mathrm{H})}=2 m / \epsilon, \quad h_{t r}^{(\mathrm{H})}=0,
$$

where $\epsilon$ denotes the spatial distance to the particle's location (this may be easily obtained by transforming the well known Coulomb-like Cartesian solution to spherical coordinates). Equation (49) now becomes

$$
\xi_{t, r}=-2 m / \epsilon .
$$

At this point we introduce standard Cartesian coordinates $t, x, y, z$, such that the particle is located at the origin $(x=y$ $=z=0$ ), and the $z$ direction coincides with the radial direction at the particle's location (namely, $x=r \sin \theta \cos \varphi, y$ $=r \sin \theta \sin \varphi$, and $z=r \cos \theta-r_{0}$ ). At the leading order in $\epsilon$, we may replace $\partial_{r}$ by the Cartesian derivative operator $\partial_{z}$. Equation (50) then becomes

$$
\xi_{t, z} \cong-2 m\left(z^{2}+\rho^{2}\right)^{-1 / 2},
$$

where $\rho^{2} \equiv x^{2}+y^{2}$. Equation (51) can now be easily integrated with respect to $z$ (with $x, y$ held fixed). We obtain

$$
\xi_{t} \cong-2 m \log \left(\frac{z}{\rho}+\sqrt{1+z^{2} / \rho^{2}}\right)+R(x, y),
$$

where $R(x, y)$ is an arbitrary function. This is the most general ( $t$-independent) solution for $\xi_{t}$.

Consider next the asymptotic form of $\xi_{t}$ as we go to the limit $x, y \rightarrow 0$ with fixed $z \neq 0$. One finds

$$
\xi_{t}(\rho \rightarrow 0) \cong \begin{cases}+2 m \ln (\rho / 2 z)+R(x, y), & z>0, \\ -2 m \ln (\rho / 2|z|)+R(x, y), & z<0 .\end{cases}
$$

By a suitable choice of the function $R(x, y)$ one may, at best, eliminate the divergence along one of the rays $z<0$ or $z$ $>0$ (by choosing $R \simeq \pm 2 m \ln \rho$, respectively), but not along both rays simultaneously. We thus arrive at the conclusion that $\xi_{t}$ unavoidably diverges logarithmically (at least) on approaching the axis $\rho=0$, along either the $z<0$ ray or the $z$ $>0$ ray (or both). Constructing now the $t x$ and ty components of the radiation-gauge metric perturbation, we find $h_{t x}^{(\mathrm{R})}=\xi_{t, x} \propto x / \rho^{2}$, and a similar expression for $h_{t y}^{(\mathrm{R})}$, as $\rho$ $\rightarrow 0$ (at either $z<0$ or $z>0$ ). Namely, the metric perturbation inevitably diverges at least along half the axis $\rho=0$.

It thus turns out that in the radiation gauge, the perturbation associated with a pointlike particle is represented by a string-like one-dimensional singularity. In particular, the radiation-gauge metric perturbation cannot be well defined in a complete neighborhood of the particle. (Compare with the harmonic or RW gauges, where the singularity is confined to the particle's location and the metric perturbation is well defined everywhere in the particle's neighborhood.) This pathological behavior-manifested already in the elementary case of a static particle in flat space-serves to demonstrate the pathological nature of the radiation gauge in the presence of point sources. As the radiation gauge seems inappropriate for representing the metric perturbation in the particle's neighborhood, it becomes rather meaningless to consider the self-force acting on the particle in that gauge.

Finally we note that although the indefiniteness of the radiation-reaction self-force was demonstrated here only for a static particle in flat space, the same indefiniteness should also occur generically for all types of orbits in Schwarzschild or Kerr spacetimes.

\section{SUMMARY AND DISCUSSION}

The main results of this manuscript are contained in Eqs. (6), (24), and (29). Equation (6) describes the gauge transformation of the gravitational self-force, given the gauge displacement vector $\xi_{\mu}$. Equation (24) describes, in a schematic manner, the extension of the MSTQW formulation for the gravitational self-force to an arbitrary gauge "G": It implies that the "correct" singular piece to be removed from the bare force in the G-gauge [the one derived directly from the G-gauge metric perturbation through Eq. (20)] is always to be calculated in the harmonic gauge, as described in the original analysis by MSTQW. By applying these results to our mode-sum regularization method (which was previously formulated only within the harmonic gauge [5]) we finally obtained Eq. (29), which describes a practical mode-sum prescription for construction of the gravitational self-force in any gauge " $G$ " (provided that the self-force has a regular, finite value in that gauge). We stress again that, since the gravitational self-force is a gauge-dependent notion, expressions like Eq. (24) or Eq. (29) for the self-force will be meaningful only when accompanied by the full information about the gauge to which they correspond. (Alternatively, one can specify the metric perturbation $h^{(\mathrm{G})}$ itself, which of course contains the full information about the gauge.)

The implementation of Eq. (29) for calculating the G-gauge self-force involves two distinct parts: (i) calculation of the bare modes of the force in the G-gauge [through Eq. (20)]; and (ii) derivation of the four vectorial regularization parameters $A^{\alpha}, B^{\alpha}, C^{\alpha}$, and $D^{\alpha}$. Our discussion concerning the gauge transformation of the self-force led us to conclude that the values of these regularization parameters do not depend on the gauge in which one calculates the self force: These parameters are always to be calculated in the harmonic gauge (using the analytic technique described in Ref. [5]). This "gauge invariance" property of the regularization parameters is demonstrated by the recent analysis by Lousto $[8,14]$, who calculated (numerically) the values of $A^{\alpha}, B^{\alpha}$, and $C^{\alpha}$ in the RW gauge, for a radial orbit on a Schwarzschild background. These numerical values appear to be in perfect agreement with the harmonic-gauge values derived analytically in Ref. [5] (in the case studied so far, of the self-force at a turning point of a radial geodesic). Also, the (zero) value obtained for the parameter $D^{\alpha}$ in the harmonic gauge [5] agrees with Lousto's result for $D^{\alpha}$ in the RWgauge (which was based on a proposed zeta-function regularization procedure [8]).

The prescription (29), as well as Eq. (24), is only appli- 
cable when the self-force admits a definite finite value in the G-gauge. Whether or not this is the case for a given gauge "G," can be decided with the help of Eq. (6): The analysis by MSTQW implies that the self-force will always have a regular finite value in the harmonic gauge (and it also tells us how to derive this value). Therefore, the G-gauge self-force would be well defined, in our approach, only if the transformation from the harmonic gauge to the G-gauge would yield - through Eq. (6) - a regular finite value for the force difference $\delta F_{\text {self }}^{\alpha}$. It is only in this case that we are able to use Eq. (29) for calculating the G-gauge self-force. Otherwise (namely, if $\delta F_{\text {self }}^{\alpha}$ diverges or is indefinite), Eq. (29) appears to be useless.

As an example, in Sec. $\mathrm{V}$ we explored the transformation from the harmonic gauge to the Regge-Wheeler gauge. We found that the RW self-force is well defined as long as strictly radial trajectories are considered. For such trajectories, Eq. (29) then provides a useful prescription for computing the RW self-force. However, this seems not to be the case for more general orbits, as we demonstrated by considering a circular orbit: Here, the transformation from the harmonic gauge yielded an indefinite value for the RW self-force. The situation is even worse in the radiation gauge, where $\delta F_{\text {self }}^{\alpha(\mathrm{H} \rightarrow \mathrm{R})}$ is found to be not only discontinuous but also unbounded, and presumably for all types of orbits.

How could one interpret a situation where $\delta F_{\text {self }}^{\alpha}$ diverges (or is indefinite)? In some occasions, such a result may be attributed to a severe pathology of the gauge. This seems to be the case in the radiation gauge, as implied by the fact that in this gauge the metric perturbation diverges not only at the particle's location, but also along an (ingoing or outgoing) radial ray emerging from the particle (see Sec. V). However, the situation seems to be different in the RW gauge, in which the metric perturbation is well-defined in the neighborhood of the particle (though of course not at the particle itself), like in the harmonic gauge. In this case we have seen that, for nonradial orbits, $\delta F_{\text {self }}^{\alpha(\mathrm{H} \rightarrow \mathrm{RW})}$ (and hence also $F_{\text {self }}^{\alpha(\mathrm{RW})}$ itself) is ill defined. This originates from the fact that certain components of $\xi^{\mu}$-e.g. $\xi^{\theta}$ or $\xi^{\varphi}$-admit a directiondependent limit (as demonstrated by the dependence of e.g. $\xi_{y}$ on $\phi$; cf. Sec. V).

This situation-a direction-dependent expression for the self-force in certain gauges-motivates one to consider a simple generalization of the standard MSTQW regularization procedure, by averaging over all spatial directions. Namely, one can evaluate the limit of the right-hand side of Eq. (24) (or, similarly, the limit of the displacement vector $\xi^{\mu}$ ) along fixed spatial null geodesics emanating from the particle, and then average over the solid angle (in the particle's rest frame). This would clearly be a generalization of the MSTQW procedure, because whenever the coincidence limit is well defined, the average over solid angle will be welldefined too, and will yield the same result. One still needs to investigate how this averaging over directions is to be implemented within the context of the mode-sum regularization.

The above generalized regularization procedure will yield a definite self-force in a wide class of gauges (though not in all gauges; obviously one can construct a displacement vec- tor $\xi^{\mu}$ which does not even have a directional limit, in which case the generalized regularization procedure will fail to yield a definite self-force). The analysis in Sec. V suggests that for circular orbits the displacement vector $\xi^{\mu}$ from the harmonic to the RW gauge may have a well-defined directional limit, and hence the RW self-force may be well defined within this generalized prescription. Recall, however, that the above analysis does not completely guarantee this regularity of the (generalized) RW self-force, because so far we have only analyzed the tangential components $\xi_{y}$ and $\xi_{z}$ (which yield $\xi^{\theta}$ and $\xi^{\varphi}$ ), but not $\xi_{t}$ and $\xi_{r}$. Also, our analysis was restricted to the surface $r=r_{0}, t=0$, i.e. to directional limits through tangential directions.

There seems to be another procedure that would allow one to use the metric perturbations in e.g. the RW or radiation gauges for useful self-force calculations (without resorting to the above generalized regularization procedure). We shall now briefly outline here a preliminary version of this procedure. (We note that a similar approach has been proposed by Mino [11].) Suppose that the metric perturbation $h^{(\mathrm{G})}$ is known (e.g., in the form of mode decomposition), where " $G$ " refers to either the RW or radiation gauges. If we knew how to convert $h^{(\mathrm{G})}$ to the harmonic gauge, it would be straightforward to construct the self-force from it, through Eqs. (19) or (25). However, performing the transformation $\mathrm{G} \rightarrow \mathrm{H}$ requires one to solve a system of partial differential equations for $\xi^{\mu}$, and unfortunately we do not know the exact solution of this system. Nevertheless, it appears possible to construct an approximate, leading-order, solution of this system, for both the RW and the radiation gauges. This was demonstrated in Sec. V (for both gauges) in a few simple cases, and it appears likely that the leading-order solution can be generalized to a generic orbit. Let us denote this leading-order solution by $\hat{\xi}^{\mu}$. In principle one can then use $\hat{\xi}^{\mu}$ to transform the metric perturbations from the original gauge $\mathrm{G}$ to an "approximate harmonic" gauge, which we denote $\hat{H}$. Presumably, in the gauge $\hat{H}$ the self-force will be well defined, since the metric perturbations in the harmonic and $\hat{\mathrm{H}}$ gauges share the same leading-order asymptotic behavior. After decomposing $\hat{\xi}^{\mu}$ into $l$-modes, one can use the mode-sum regularization method to calculate the $\hat{\mathrm{H}}$-gauge self-force: Applying Eq. (23) for each of the single $l$-modes, with " $\mathrm{H}$ " and "G" replaced, correspondingly, by " $G$ " and "Ĥ," we first get

$$
F_{\text {bare }}^{\alpha l(\hat{\mathrm{H}})}=F_{\text {bare }}^{\alpha l(\mathrm{G})}+\delta F_{\text {self }}^{\alpha l(\mathrm{G} \rightarrow \hat{\mathrm{H}})},
$$

where $\delta F_{\text {self }}^{\alpha l(\mathrm{G} \rightarrow \hat{\mathrm{H}})}$ is to be obtained from Eq. (6) by replacing $\xi^{\lambda}$ by the l-mode of $\hat{\xi}^{\mu}$. Then, writing Eq. (29) for the H-gauge (i.e., with all "G" replaced by " $\hat{\mathrm{H}}$ ") and substituting the above expression for $F_{\text {bare }}^{\alpha l(\hat{\mathrm{H}})}$, one obtains 


$$
\begin{aligned}
F_{\text {self }}^{\alpha(\hat{\mathrm{H}})}= & \sum_{l=0}^{\infty}\left[\left(F_{\text {bare }}^{\alpha l(\mathrm{G})}+\delta F_{\text {self }}^{\alpha l(\mathrm{G} \rightarrow \hat{\mathrm{H}})}\right)-A^{\alpha} L-B^{\alpha}-C^{\alpha} / L\right] \\
& -D^{\alpha}
\end{aligned}
$$

which provides a prescription for calculating the $\hat{\mathrm{H}}$-gauge self-force through the modes of the bare force in the $G$ gauge. We hope to further develop and implement this method elsewhere.

\section{ACKNOWLEDGMENTS}

It is our pleasure to thank Eric Poisson, Carlos Lousto, and Yasushi Mino for commenting on an early version of this manuscript, and for valuable discussions. This research was supported in part by the United-States-Israel Binational Science Foundation. L.B. was also supported by the European Community program IHP-MCIF-99-1 under contract number HPMF-CT-2000-00851.
[1] Y. Mino, M. Sasaki, and T. Tanaka, Phys. Rev. D 55, 3457 (1997).

[2] T. C. Quinn and R. M. Wald, Phys. Rev. D 56, 3381 (1997).

[3] Laser Interferometer Space Antenna, Proceedings of the Second International LISA Symposium, edited by William M. Folkner, AIP Conf. Proc. No. 456 (American Institute of Physics, Woodbury, NY, 1998).

[4] M. J. Pfenning and E. Poisson, gr-qc/0012057.

[5] L. Barack, Phys. Rev. D 64, 084021 (2001).

[6] L. Barack and A. Ori, Phys. Rev. D 61, 061502(R) (2000); L. Barack, ibid. 62, 084027 (2000).

[7] L. M. Burko, Class. Quantum Grav. 17, 227 (2000); Phys. Rev. Lett. 84, 4529 (2000); L. Barack and L. M. Burko, Phys. Rev. D 62, 084040 (2000); L. M. Burko, Y.-T. Liu, and Y. Soen, ibid. 63, 024015 (2001); L. M. Burko and Y.-T. Liu, ibid. 64, 024006 (2001).

[8] C. O. Lousto, Phys. Rev. Lett. 84, 5251 (2000).

[9] T. Regge and J. A. Wheeler, Phys. Rev. 108, 1063 (1957).

[10] F. J. Zerilli, J. Math. Phys. 11, 2203 (1970); Phys. Rev. D 2 , 2141 (1970).
[11] Y. Mino, a talk given in the fourth "Capra Ranch Meeting on Radiation Reaction” (May 2001, Golm)—see http://www.aeipotsdam.mpg. de/ lousto/ capra/ proceedings/ Mino/Mino.html. Some preliminary ideas concerning gauge transformations of the self-force were also presented in Y. Mino and H. Nakano, Prog. Theor. Phys. 100, 507 (1998); Y. Mino, in Proceedings of the Tenth Workshop on General Relativity and Gravitation in Japan, edited by M. Sasaki, J. Yokoyama, T. Nakamura, and K. Tomita, 2000; see also http://www.tapir.caltech.edu/capra3/ Proceedings/mino/index.html

[12] P. L. Chrzanowski, Phys. Rev. D 11, 2042 (1975).

[13] C. W. Misner, K. S. Thorne, and J. A. Wheeler, Gravitation (Freeman, San Francisco, 1970).

[14] L. Barack and C. O. Lousto (in preparation); some preliminary results of this work are given in C. O. Lousto, "Proceedings of the 3rd International LISA Symposium," gr-qc/0010007.

[15] For the values of $u_{\varphi}$ and $u_{t}$ in circular orbits see, e.g., S. Chandrasekhar, The Mathematical Theory of Black Holes (Oxford University Press, New York, 1983), Chap. 3, Eq. (140). 